Artículo científico

(Original paper)

\title{
VARIACIÓN DE LA DIVERSIDAD DE STAPHYLINIDAE, SILPHIDAE Y TROGIDAE (INSECTA: COLEOPTERA) EN UN GRADIENTE URBANO-AGRÍCOLA EN UNA REGIÓN SEMIÁRIDA DEL ESTADO DE MÉXICO, MÉXICO
}

\author{
VARIATION OF THE DIVERSITY OF STAPHYLINIDAE, SILPHIDAE AND TROGIDAE \\ (INSECTA: COLEOPTERA) IN A URBAN-AGRICULTURAL GRADIENT IN A SEMIARID \\ REGION OF ESTADO DE MEXICO, MEXICO
}

\author{
ESTEBAN JIMÉNEZ-SÁNCHEZ ${ }^{*}$, ROBERTO QUEZADA-GARCÍA ${ }^{2}$, JORGE PADILLA-RAMÍREZ ${ }^{1}$, \\ MARICEla L. MORENO ${ }^{1}$, MóniCa A. ANGEL ${ }^{1}$ \\ ${ }^{1}$ Laboratorio de Zoología, Facultad de Estudios Superiores Iztacala, Universidad Nacional Autónoma de México. Av. de los \\ Barrios 1, Los Reyes Iztacala, 54090, Tlalnepantla, Estado de México, México. <estjimsan@gmail.com>; \\ <jorgepr6@gmail.com>; <mariloxos@gmail.com>; <monyantr13@hotmail.com> \\ ${ }^{2}$ Centre d'Étude de la Forêt (CEF) y Département des Sciences du Bois et de la Forêt, Faculté de Foresterie, Géographie et \\ Géomatique, Université Laval, Canadá. <roberto_queza71@ @otmail.com> \\ *Autor de correspondencia: <estjimsan@gmail.com>
}

Recibido: 29/11/2018; aceptado: 28/11/2019; publicado en línea: 13/12/2019

Editor responsable: Carmen Huerta Crespo

Jiménez-Sánchez, E., Quezada-García, R., Padilla-Ramírez, J. R., Moreno, M. L., Angel, M. A. (2019) Variación de la diversidad de Staphylinidae, Silphidae y Trogidae (Insecta: Coleoptera) en un gradiente urbano-agrícola en una región semiárida del Estado de México, México. Acta Zoológica Mexicana (nueva serie), 35, 1-16. https://doi.org/10.21829/azm.2019.3502152

RESUMEN. Se estudió el efecto del uso de suelo sobre la diversidad de coleópteros necrófilos y epigeos en una zona semiárida del Estado de México, poniendo a prueba la siguiente hipótesis: las áreas dedicadas a la producción agrícola representan un refugio para las especies de Staphylinidae, Trogidae y Silphidae. Para lo cual se realizaron muestreos mensuales durante un año con trampas de caída y trampas tipo NTP-80 cebadas con carroña. Ambas trampas fueron distribuidas en tres localidades con diferente uso de suelo (zona agrícola, zona suburbana y zona urbana). En la zona agrícola la abundancia y riqueza fueron mayores, y la diversidad fue significativamente menor que lo registrado en la zona suburbana y urbana, debido a que en la zona agrícola dos especies (Nicrophorus mexicanus Mattews, 1888 y Belonuchus sp.) concentraron el $70 \%$ de la abundancia, mientras que en las otras dos zonas las especies tuvieron mayor equidad. El recambio espacial de especies y el anidamiento contribuyeron de manera similar en la variación de la composición del ensamble de especies entre los sitios, la zona agrícola albergó el 70.6\% de las especies que estuvieron presentes tanto en la zona urbana como en la zona suburbana o en alguna de éstas, así como un mayor número de especies con afinidad alta por este sitio, lo que sugiere que la zona agrícola funciona como refugio de las especies de coleópteros necrófilos y epigeos que se distribuyen en esta región semiárida, cuyas poblaciones se vieron favorecidas durante todo el año, debido al aporte constante de materia orgánica y humedad. Finalmente, en cuanto al inventario de especies, se registró a Belonuchus erichsoni Bernhauer, 1917 por primera vez para el Estado de México.

Palabras clave: coleópteros; necrófilos; epigeos; matorral xerófilo; Teotihuacan; NTP-80; trampa de caída 
Jiménez-Sánchez, E., Quezada-García, R., Padilla-Ramírez, J. R., Moreno, M. L., Angel, M. A. (2019) Variation of the diversity of Staphylinidae, Silphidae and Trogidae (Insecta: Coleoptera) in a urbanagricultural gradient in a semiarid region of Estado de México, Mexico. Acta Zoológica Mexicana (nueva serie), 35, 1-16. https://doi.org/10.21829/azm.2019.3502152

\begin{abstract}
The effect of land use on the diversity of necrophilous and epigeal beetles in a semi-arid zone of the Estado de México was studied, we tested the following hypothesis: areas dedicated to agricultural production represent a refuge for species of Staphylinidae, Trogidae and Silphidae. Monthly sampling was carried out during one year with pitfall and carrion traps. Traps were distributed in three locations with different land use (agricultural, suburban and urban area). In the agricultural zone the abundance and richness were greater, but the diversity was significantly less than registered in the suburban and urban area, because two species (Nicrophorus mexicanus Mattews, 1888 and Belonuchus sp.) concentrated 70\% of abundance in the agricultural area, while in the other two areas the species had greater evenness. Spatial species turnover and nestedness contributed equally to the variation in the composition of the species assemblages between sites, the agricultural zone harbored $70.6 \%$ of the species that were present both in the urban and in the suburban area or in any of these, also they have the greater number of species with high affinity for this site. The results suggest that the agricultural area functions as a refuge for the necrophilous and epigeal beetle species that are distributed in this semi-arid region, whose populations were favored throughout the year due to the constant contribution of organic matter and moisture. Finally, regarding the inventory of species, Belonuchus erichsoni Bernhauer, 1917 was registered for the first time for the Estado de México.
\end{abstract}

Key words: beetles; necrophilous; epigean; xeric shrub; Teotihuacan; NTP-80; pitfall

\title{
INTRODUCCIÓN
}

A nivel mundial los seres humanos han alterado en varios grados casi la mitad de la superficie terrestre, la vegetación natural ha sido reemplazada por áreas urbanas, campos agrícolas y el pastoreo de ganado, las ciudades crecen $2 \%$ cada año, de manera que las poblaciones urbanas, de acuerdo con la división de población de las Naciones Unidas, alcanzarán $60 \%$ del total mundial para el año 2030. Aunque estas áreas pueden ser un hábitat para algunas especies que habitaron en los ecosistemas naturales, la mayoría solo puede sobrevivir en áreas limitadas de la vegetación original, donde satisfacen sus necesidades de temperatura, agua y alimento, y en ningún otro lado. De hecho, de acuerdo con la lista roja de 2006 de la IUCN, la limpieza de bosques para cultivo y pastoreo es una amenaza para más del $20 \%$ de las especies terrestres (Chivian \& Bernstein, 2015).

La zona de estudio está ubicada en los municipios de Teotihuacán y San Martín de las Pirámides, Estado de México, donde alrededor del 70\% del suelo es de uso agrícola, 16\% zona urbana, y únicamente 14\% corresponde a la vegetación de matorral y pastizal (INEGI, 2010a, b). Es indudable que estas alteraciones han conducido a la pérdida de biodiversidad y a la invasión de especies exóticas; sin embargo, se desconoce el estado actual de las poblaciones de coleópteros.

Los coleópteros han sido empleados comúnmente en monitoreos biológicos, tal es el caso de las familias Carabidae y Staphylinidae, que incluyen principalmente especies depredadoras, debido a que son muy diversos, abundantes y bien conocidos, esto último, principalmente en Europa y Norte América. Los coleópteros epigeos de estas familias en bosques fragmentados revelaron que los estafilínidos fueron más sensibles que los carábidos a los efectos de la urbanización; aunque la riqueza no declina de acuerdo con un gradiente rural-urbano, la proporción de estafilínidos especialistas de los bosques disminuye con el incremento de la urbanización y favorece a las especies especialistas de los hábitats abiertos (Deischel, 2006). Sin embargo, Ishitani et al. (2003) encontraron que tanto la abundancia como la riqueza de carábidos 
decrecen significativamente con el incremento de la urbanización, y corroboraron la idea de que las áreas perturbadas están caracterizadas por especies de tallas pequeñas y generalistas.

Con respecto a la comunidad de coleópteros que se alimentan de carroña, como es el caso de la familia Silphidae, se ha visto que la diversidad de sílfidos a lo largo de un gradiente urbano-rural decrece en función de la pérdida y fragmentación del bosque (Wolf \& Gibbs, 2004). Por lo tanto, los sílfidos son más exitosos en bosques extensos que en los pequeños fragmentos generados por la urbanización (Trumbo \& Bloch, 2000). Asimismo, los escarabeidos (Scarabaeinae) han sido sugeridos como potenciales bioindicadores en las regiones tropicales, por su sensibilidad a los cambios en el hábitat (Arellano et al., 2005; Nichols et al., 2007; Arellano \& Castillo-Guevara, 2014). Para la familia Trogidae, se tiene poca información sobre la forma en que sus especies son afectadas por la perturbación, son consideradas de hábitos telionecrófagos (consume cadáveres secos y restos de pelos y plumas) y su mayor riqueza se presenta en los bosques tropicales y en los ambientes xéricos (Morón, 2003).

En México los grupos que han recibido mayor atención desde el punto de vista taxonómico y ecológico, son los coleópteros de las familias Scarabaeidae (Scarabaeinae) y Silphidae. Por el contrario, para la familia Staphylinidae, aunque en los últimos años se ha incrementado su conocimiento, aún hace falta trabajo taxonómico que permita conocer mejor la diversidad de estafilínidos. Los estudios sobre este grupo han sido llevados a cabo en gradientes o en mosaicos de diferentes tipos de vegetación (Márquez, 2003; Caballero et al., 2009; Jiménez-Sánchez et al., 2011; Caballero \& León-Cortés, 2012; JiménezSánchez et al., 2013; Rodríguez et al., 2019) y han arrojado datos importantes sobre la composición de los ensambles y los cambios asociados con la perturbación o con el manejo del suelo que induce un cambio de la cubierta terrestre, lo que ha permitido tentativamente correlacionar las diferencias de la riqueza y composición de especies con las características del hábitat, así como aumentar el conocimiento para algunas especies de las determinantes ambientales, hábitos alimentarios, altitud, entre otros aspectos que influyen en su distribución. Por lo tanto, en el presente estudio se investiga como el tipo de uso de suelo determina la abundancia, riqueza y diversidad de las familias de coleópteros necrófilos y epígeos (Staphylinidae, Silphidae y Trogidae) en un gradiente urbano-agrícola. Se puso a prueba la siguiente hipótesis: el uso del suelo con fines agrícolas representa un refugio para las especies, comparado con un sitio de asentamiento urbano y uno semiurbano.

\section{MATERIALES Y MÉTODOS}

Área de estudio. La zona se encuentra en el centro de México, al nororiente del Estado de México, en los municipios de San Martín de las Pirámides y San Juan Teotihuacán, a una altitud promedio de 2,300 m (Fig. 1). El principal uso del suelo es la agricultura de temporal y de riego que abarca el $77 \%$ del territorio, $16.5 \%$ corresponde con la zona urbana y solo el 6.5\% lo ocupan el matorral y el pastizal (INEGI, 2010a, b). El clima es semiseco (INEGI, 2017), la temperatura media anual es de $14.1^{\circ} \mathrm{C}$, la temporada de lluvias va de mayo a octubre $(>43 \mathrm{~mm}$ ) y la de sequía de noviembre a abril, la precipitación total anual es de $515.2 \mathrm{~mm}$ de acuerdo con los registros de la estación meteorológica Otumba (Clave 15065) (SMN, 2017).

Sitios de muestreo. Los muestreos se llevaron a cabo en tres localidades con uso de suelo agrícola, suburbano y urbano (Fig. 1). En la zona agrícola se práctica la agricultura de riego anual y semipermanente con un tipo de suelo feozem de textura media (INEGI, 2018). De manera particular, el punto de muestreo se ubicó en un cultivo de Opuntia ficus-indica (L.) Mill. [19 40' 37.4" N, 98 48' 59.2" O]. Esta localidad estuvo sujeta a un manejo agrario que consistió en poda, fumigación y quema, con el fin de maximizar la producción de tuna y los cultivos de Zea mays L., Phaseolus vulgaris L., Medicago sativa L. y Beta vulgaris (L.) K.Koch establecidos en los alrededores (com. pers. agricultores locales). 


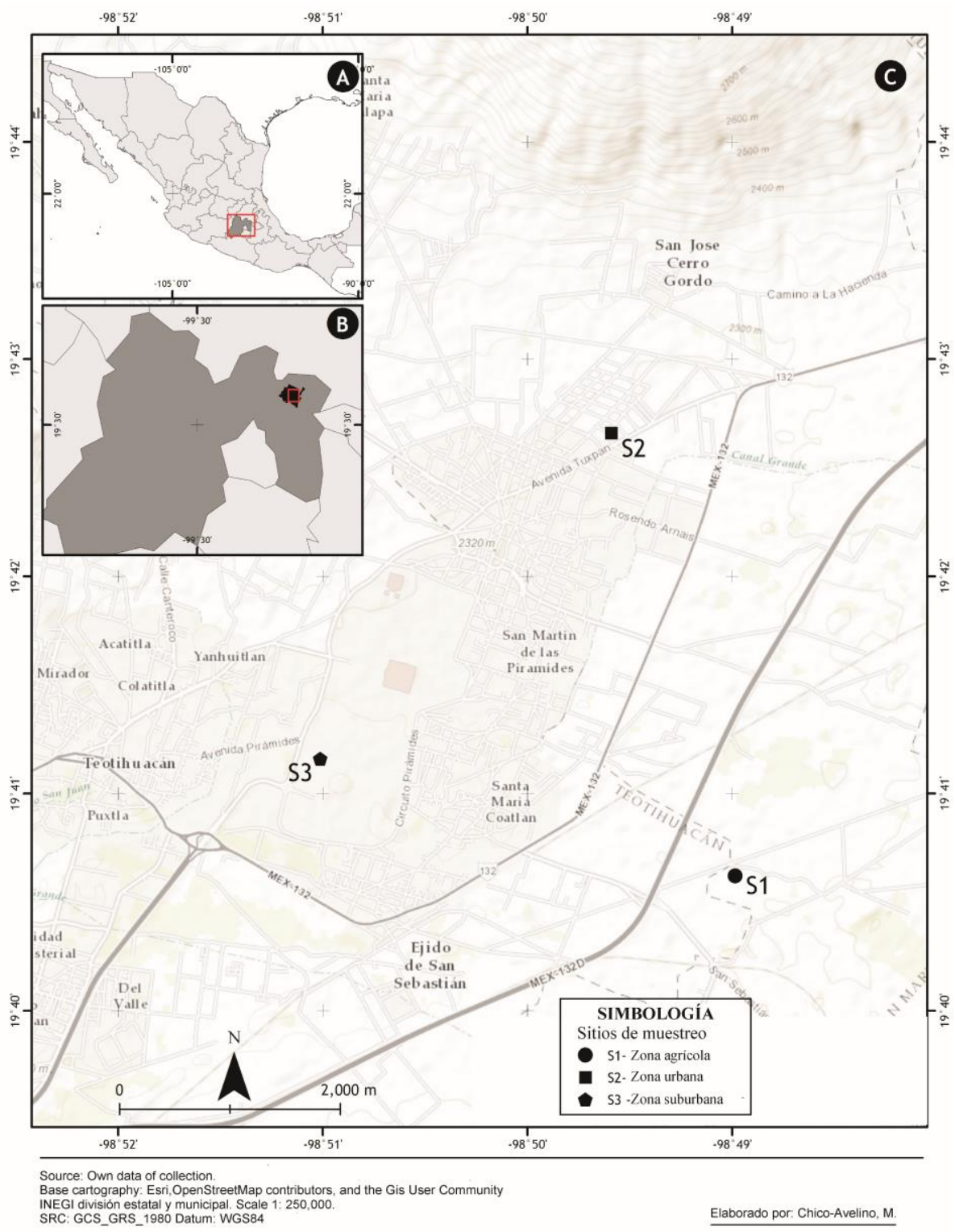

Figura 1. Área de estudio. Nivel nacional (A), nivel estatal (B) y sitios de recolecta (C).

La zona suburbana correspondió a la zona arqueológica de Teotihuacán [19 $49^{\circ} 41^{\prime} 11^{\prime \prime} \mathrm{N}, 98^{\circ} 51^{\prime}$ 00.9" O], el tipo de suelo correspondió al tipo feozem, secundario durisol, de textura media, donde predominó el matorral constituido de: Bouvardia ternifolia (Cav.) Schltdl., Brickellia veronicifolia (Kunth) A. Gray, Mentzelia hispida Willd., Mimosa aculeaticarpa var. biuncifera (Benth.) Barneby, Montanoa tomentosa Cerv., Opuntia hyptiacantha F.A.C. Weber, Opuntia streptacantha Lem., Schinus molle y Senecio praecox (Cav.) DC.; también se presentó el pastizal, compuesto por varias especies de gramíneas y plantas herbáceas en los grandes espacios de la zona que están representadas por: Euphorbia hirta L., Pseudognaphalium arizonicum (A. Gray) Anderb., Erodium cicutarium (L.) L'Hér. ex Aiton, Lepidium virginicum L., Conyza canadensis (L.) Cronquist, Conyza coronopifolia Kunth, Conyza sophiifolia Kunth, Sisymbrium officinale (L.) Scop., Verbena bipinnatifida Nutt., Oxalis corniculata L., Gaura coccinea Nutt. ex Pursh, Reseda luteola L. y Taraxacum officinale F.H. Wigg. (Torres, 2001). La vegetación mencionada estuvo afectada por las actividades de mantenimiento de los monumentos y de los alrededores, que consistió en el retiro de la materia orgánica y quemas periódicas. 
En la zona urbana [ $19^{\circ} 42^{\prime} 40.4^{\prime \prime} \mathrm{N}, 98^{\circ} 49^{\prime} 35.5^{\prime \prime}$ O] (INEGI, 2018) los muestreos se realizaron en el patio de dos casas, donde predominó la vegetación que comprendió a diversas especies ornamentales, cultivadas, arvenses y ruderales como: Opuntia ficus-indica, Agave salmiana Otto ex Salm-Dyck, Ficus carica L., Schinus molle L., Amaranthus sp, Curcubita pepo L., Chenopodium nuttallie Saff., Tithonia tubiformis (Jacq.) Cass., Simsia amplexicaulis (Cav.) Pers., Bidens pilosa L. y Sanvitalia procumbens Lam., entre otras.

Trabajo de campo. En cada sitio se colocaron cuatro trampas tipo NTP-80 (Morón \& Terrón, 1984), cebadas con calamar para la captura de coleópteros necrófilos y diez trampas de caída (pitfall) sin cebo para las especies epígeas, estas últimas consistieron en vasos de plástico de $1 \mathrm{~L}(14.5 \mathrm{~cm}$ x $11 \mathrm{~cm})$, mismas que se enterraron a ras de suelo y se cubrieron con un plato a $5 \mathrm{~cm}$ de altura del borde superior del vaso para evitar su inundación; en ambos tipos de trampa se empleó monoetilen-glicol como líquido conservador. Las trampas fueron distribuidas a lo largo de un transecto longitudinal, entre cada trampa NTP-80 hubo una separación aproximada de $100 \mathrm{~m}$ (con excepción de las instaladas en la zona urbana donde la separación entre trampa fue de aproximadamente $50 \mathrm{~m}$ ) y entre cada trampa de caída de $20 \mathrm{~m}$. Las muestras fueron recuperadas mensualmente de enero a diciembre de 2012. Los ejemplares obtenidos fueron colocados en frascos de plástico con etanol al 70\% y transportados al Laboratorio de Zoología de la FES-Iztacala, UNAM.

Trabajo de laboratorio. Los ejemplares se separaron y se determinaron mediante el uso de las claves taxonómicas de Smetana (1995) y Navarrete-Heredia et al. (2002) para la familia Staphylinidae; de Navarrete-Heredia (2009) para la familia Silphidae y de Vaurie (1955) para la familia Trogidae. Además, los especímenes fueron comparados con aquellos previamente identificados de la Colección de Artrópodos de la Facultad de Estudios Superiores Iztacala (CAFESI), UNAM, Tlalnepantla, Estado de México, México, donde se depositaron los ejemplares. Las especies de Staphylinidae fueron agrupadas en gremios tróficos: depredadoras (Paederinae, Pselaphinae, Scydmaeninae, Staphylininae), micetófagas (Scaphidiinae, Tachyporinae) y saprófagas (Oxytelinae) de acuerdo con Thayer (2005) y Jałoszynski (2018). Las especies de Silphidae en necrófagas y las de Trogidae como telionecrófagas de acuerdo con Deloya et al. (2007). La subfamilia Aleocharinae (Staphylinidae) fue excluida del análisis debido a su dificultad taxonómica.

Tratamiento estadístico. Para evaluar las diferencias entre los componentes de la diversidad alfa y la eficiencia del muestreo en los sitios, se empleó el método propuesto por Chao et al. (2010). Para ello, se obtuvieron los datos de abundancia de las especies para los tres sitios y la estructura cuantitativa de la comunidad se evaluó a través de curvas de rango-abundancia, elaboradas a partir del logaritmo base diez del valor de abundancia de cada especie. Para cada sitio se elaboraron las curvas integradas de extrapolación y rarefacción para los números de Hill (riqueza de especies $(\mathrm{q}=0)$, diversidad de Shannon $(\mathrm{q}=1)$, concentración inversa de Simpson $(q=2)$, con un intervalo de confianza de 95\%, con base en el método de Bootstrap (nboots $=50$ ), para ello se extrapoló duplicando el número de individuos de cada sitio. Para comparar los valores de diversidad entre los sitios, se hicieron las curvas de extrapolación y rarefacción basados en el tamaño de la muestra para cada valor fijo de q con $95 \%$ de intervalo de confianza, previamente se calculó el tamaño de muestra base.

Para saber que tan completo fue el muestro para cada sitio, se construyó una curva de completitud de la muestra; posteriormente, se graficaron las curvas de extrapolación y rarefacción basados en la cobertura de la muestra para la diversidad. Finalmente, se compararon las diversidades de cada uno de los sitios basadas en la cobertura de la muestra para cada valor de q, previo cálculo de la cobertura base. Los análisis se realizaron con la paquetería Inext 2.0.19 (Hsieh et al., 2016). Para analizar la disimilitud entre los sitios y meses, se utilizó el método propuesto por Baselga (2010) que desglosa la diversidad beta ( $\left.\beta_{\text {SOR }}\right)$ en sus componentes aditivos, el recambio espacial de especies $\left(\beta_{\mathrm{SIM}}\right)$ y el anidamiento de los ensambles $\left(\beta_{\mathrm{NES}}\right)$. Estos análisis fueron realizados con la paquetería betapart Baselga (2010). Los gremios tróficos fueron analizados por medio de una prueba de $X^{2}$. Por último, para conocer la asociación de las especies con el uso de suelo, se determinaron las especies indicadoras con la paquetería labdsv (Roberts, 2012) a través 
de un análisis de asociación presencia-ausencia entre cada sitio. Todos los análisis fueron llevados a cabo con el programa estadístico R Development Core Team, versión. 3.5.2. (2019).

\section{RESULTADOS}

Se obtuvieron un total de 34 especies y 1,258 individuos. La familia Staphylinidae tuvo la mayor riqueza (s = 29) y abundancia $(\mathrm{n}=646)$ seguida de Silphidae $(\mathrm{s}=4, \mathrm{n}=601)$ y Trogidae $(\mathrm{s}=1, \mathrm{n}=11)$ (Cuadro 1). Belonuchus erichsoni Bernhauer, 1917 (Staphylinidae) se registra por primera vez para el Estado de México. La riqueza de especies y la abundancia fueron mayores en la zona agrícola (29 especies y 785 individuos) que en la zona suburbana y urbana, las cuales tuvieron 22 especies cada una, con 345 y 128 individuos respectivamente. Por el contrario, la diversidad de los órdenes $\mathrm{q}=1$ y $\mathrm{q}=2$ fue mayor en la zona urbana (9.59 y 5.41, respectivamente), seguida de la zona suburbana (8.39 y 5.39) y disminuyó en la transición hacia la zona agrícola (6.2 y 3.1), esta última fue la única que mostró diferencias significativas (Fig. 2). Las curvas de rango abundancia de la zona agrícola y la zona suburbana mostraron una proporción menor de especies con menos de diez individuos (61\% y 73\%, respectivamente) que la zona urbana, donde el $90 \%$ fueron raras, pero fue donde las especies más comunes tuvieron mayor equidad que las especies más comunes de los otros dos sitios (Fig. 3).
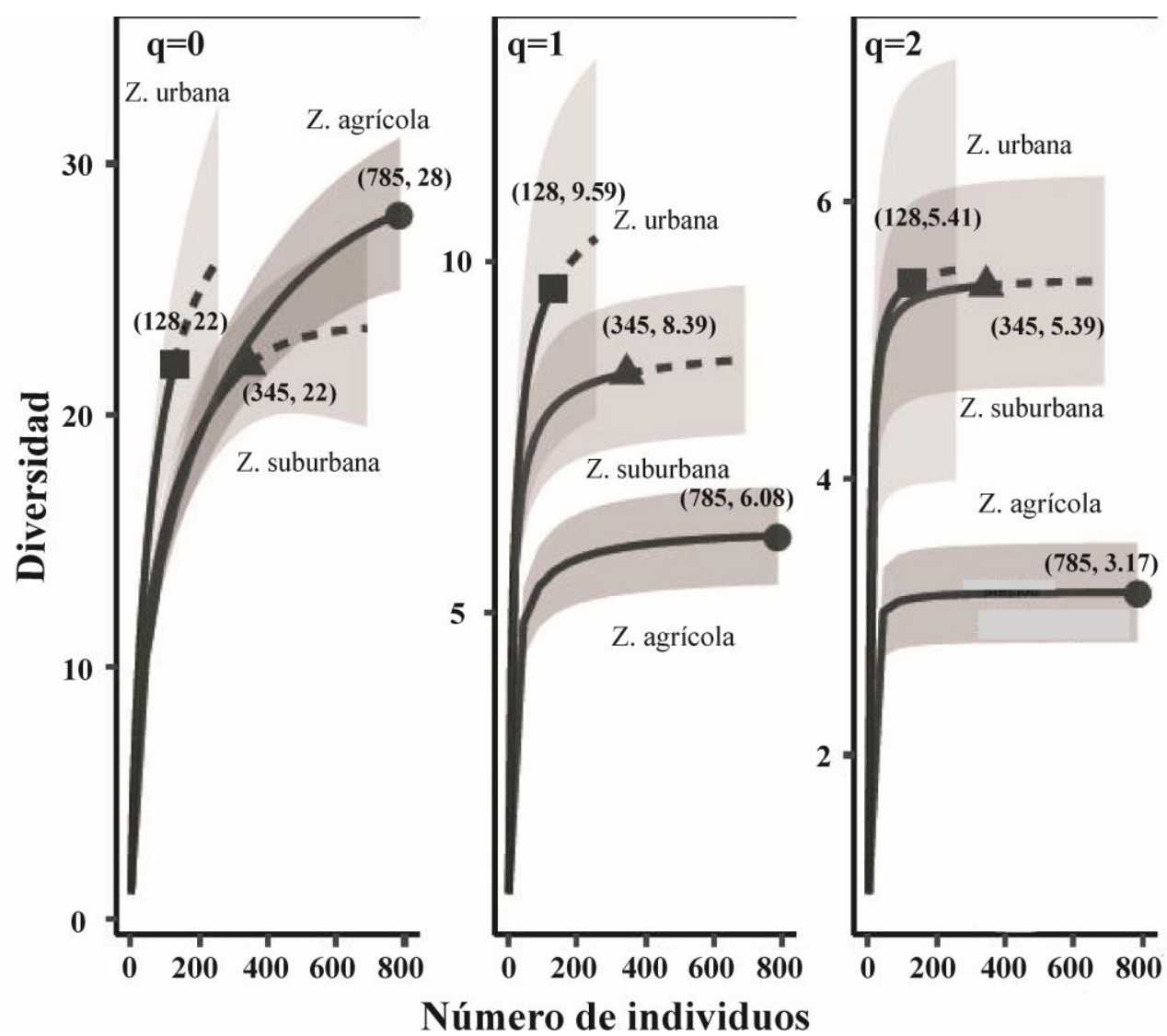

Figura 2. Comparación de las curvas de rarefacción (línea continua) y extrapolación (línea discontinua) basadas en el tamaño de la muestra de la diversidad de especies de coleópteros para los números de Hill del orden $\mathrm{q}=0$ (izquierda), $\mathrm{q}=1$ (centro) y q $=2$ (derecha). Las muestras de referencia para cada sitio se indican con figuras: zona urbana (cuadro), zona suburbana (triángulo) y zona agrícola (círculo). Los números en paréntesis son el tamaño de la muestra y los números de Hill observados para cada muestra de referencia. 
Cuadro 1. Lista de especies y su abundancia por sitio y tipo de método de recolecta. Las especies exclusivas del sitio están marcadas con un asterisco. NE. Necrófago. TNE. Telio-necrófago. SA. Saprófago. PR. Depredador. MI. Micetófago.

\begin{tabular}{llcllll} 
& & \multicolumn{2}{c}{ Zona agrícola } & Zona suburbana & Zona urbana & Total \\
TAXA Hábitos & NTP-80 & Pitfall & NTP-80 & Pitfall & NTP-80 Pitfall & general
\end{tabular}

\begin{tabular}{|c|c|c|c|c|c|c|c|c|}
\hline Silphidae & & & & & & & & \\
\hline 1. Nicrophorus mexicanus Mattews, 1888 & NE & 412 & - & 110 & - & 3 & - & 525 \\
\hline 2. Thanatophilus truncatus (Say, 1823) & NE & 26 & - & 32 & - & 2 & - & 60 \\
\hline 3. Thanatophilus graniger (Chevrolat, 1883 ) & $\mathrm{NE}$ & 12 & - & 2 & - & - & - & 14 \\
\hline 4. Oxelytrum discicolle (Brullé, 1840) & NE & - & - & $2 *$ & - & - & - & 2 \\
\hline Trogidae & & & & & & & - & \\
\hline 5. Trox plicatus Robinson, 1940 & TNE & 5 & - & 5 & - & 1 & - & 11 \\
\hline Staphylinidae & & & & & & & & \\
\hline Oxytelinae & & & & & & & & \\
\hline 6. Anotylus sp. & SA & 1 & 1 & 3 & - & 14 & 14 & 33 \\
\hline 7. Apocellus sp. & SA & - & - & - & - & - & $2 *$ & 2 \\
\hline Paederinae & & & & & & & & \\
\hline 8. Deroderus sp. & PR & - & 1 & - & 1 & - & - & 2 \\
\hline 9. Echiaster sp. & PR & 2 & 1 & 7 & - & - & - & 10 \\
\hline 10. Suniocharis sp. & PR & - & $1 *$ & - & - & - & - & 1 \\
\hline Pselaphinae & & & & & & & & \\
\hline 11. Pselaphinae sp.1 & PR & - & 2 & - & 7 & 1 & 2 & 12 \\
\hline 12. Pselaphinae sp. 2 & PR & - & $2 *$ & - & - & - & - & 2 \\
\hline 13. Pselaphinae sp.3 & PR & - & - & - & - & - & $1 *$ & $\overline{1}$ \\
\hline Scaphiidinae & & & & & & & & \\
\hline 14. Baeocera sp. 1 & MI & - & 26 & - & 15 & - & 7 & 48 \\
\hline 15. Baeocera sp. 2 & MI & - & 7 & - & 2 & - & 4 & 13 \\
\hline Scydmaeninae & & & & & & & & \\
\hline 16. Scydmaeninae sp. 1 & PR & - & 10 & - & 2 & - & 2 & 14 \\
\hline 17. Scydmaeninae sp. 2 & PR & - & - & - & - & - & $4 *$ & 4 \\
\hline 18. Scydmaeninae sp.3 & PR & - & $4 *$ & - & - & - & - & 4 \\
\hline 19. Scydmaeninae sp.4 & $\mathrm{PR}$ & - & 2 & - & 4 & - & - & 6 \\
\hline Staphylininae & & & & & & & & \\
\hline 20. Belonuchus erichsoni Bernhauer, 1917 & PR & 41 & 1 & 5 & 3 & 4 & 2 & 56 \\
\hline 21. Belonuchus ephippiatus (Say, 1830) & PR & 16 & 14 & 16 & 18 & - & - & 64 \\
\hline 22. Belonuchus sp. & PR & 122 & 16 & 68 & 16 & 37 & 8 & 267 \\
\hline 23. Creophilus maxillosus (Linnaeus, 1758) & PR & 12 & - & 8 & - & 3 & - & 23 \\
\hline 24. Heterothops sp. & PR & - & 2 & 2 & - & - & 1 & 5 \\
\hline 25. Neohypnus sp. & PR & - & 3 & 1 & - & 2 & 3 & 9 \\
\hline 26. Philonthus sp. 1 & PR & 21 & - & 14 & - & 1 & 1 & 37 \\
\hline 27. Philonthus sp. 2 & PR & $2 *$ & - & - & - & - & - & 2 \\
\hline 28. Philonthus sp. 3 & PR & 1 & - & - & - & - & 1 & 2 \\
\hline 29. Philonthus sp. 4 & PR & - & 2 & - & - & - & 1 & 3 \\
\hline 30. Philonthus sp. 5 & PR & 2 & 2 & - & - & - & 1 & 5 \\
\hline 31. Philonthus sp. 6 & PR & - & - & $1 *$ & - & - & - & 1 \\
\hline 32. Platydracus sp. & PR & - & 1 & 1 & - & - & - & 2 \\
\hline Tachyporinae & & & & & & & & \\
\hline 33. Bryoporus sp. & MI & - & 12 & - & - & 1 & 4 & 17 \\
\hline 34. Coproporus sp. & MI & - & - & - & - & $1 *$ & - & 1 \\
\hline Abundancia total & & 675 & 110 & 277 & 68 & 70 & 58 & 1258 \\
\hline Riqueza total & & 14 & 20 & 16 & 9 & 12 & 17 & 34 \\
\hline
\end{tabular}

La cobertura de la muestra estimada más alta fue para la zona agrícola con $99.5 \%$, seguida de la zona suburbana (98.8\%) y la zona urbana (94.6\%), lo que indica que el muestreo fue casi completo para las dos primeras (Fig. 4).

Los sílfidos y los trógidos fueron más abundantes en la zona agrícola (ZA) (455) y decrecieron en la transición hacia la zona suburbana (ZS) (151) y la zona urbana (ZU) (6); los estafilínidos mostraron el mismo patrón ZA (330) > ZS (194) > ZU (122); sin embargo, en cuanto a la riqueza de especies, la secuencia fue distinta, para los estafilínidos ZA (24) > ZU (19) > ZS (17), y para los otros dos grupos en conjunto fue ZS (5) > ZA (4) > ZU (2) (Cuadro 1). 


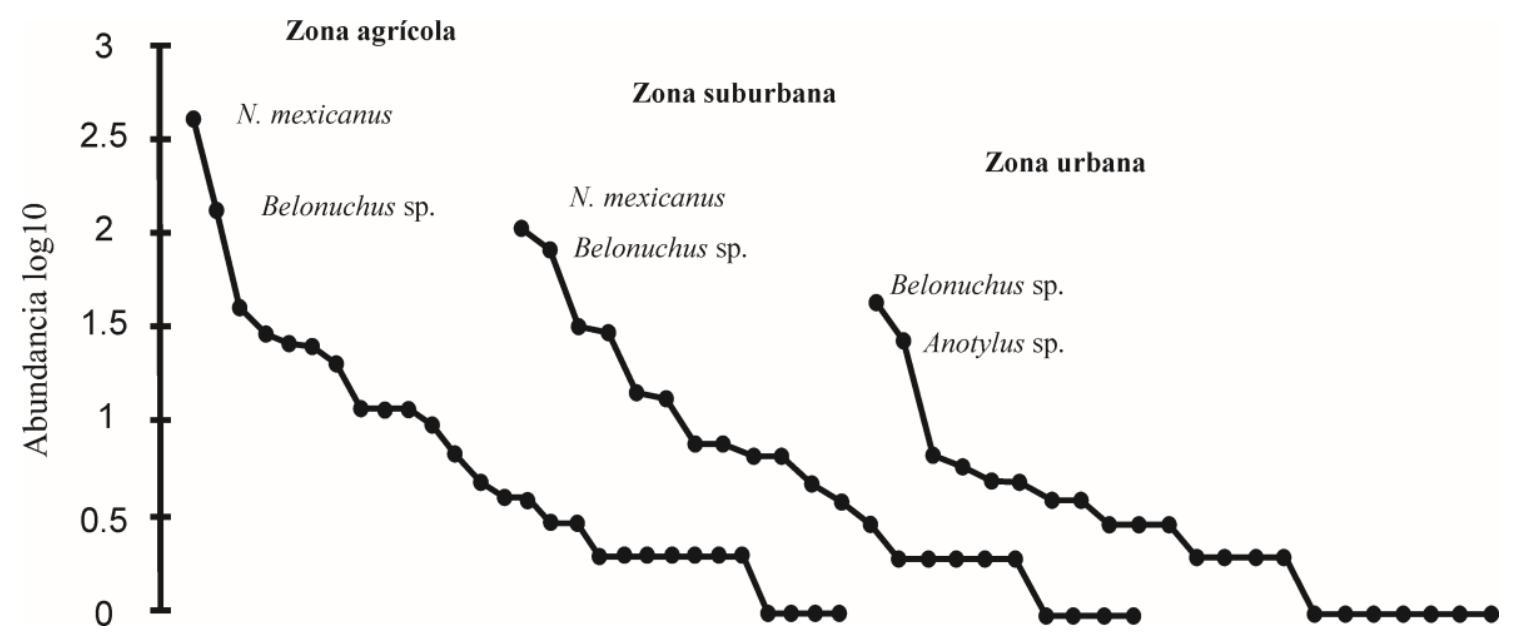

Figura 3. Curvas de rango abundancia de las especies capturadas en tres zonas.

La zona agrícola y la zona urbana tuvieron el mayor número de especies de coleópteros epigeos, mientras que en la zona suburbana predominaron las especies necrófilas (Cuadro 1). Veintidós especies y 1,022 individuos se capturaron con la trampa NTP-80, mientras que 25 especies y 236 individuos con la trampa de caída. De las tres familias estudiadas, en la trampa de caída solo se capturaron individuos de Staphylinidae, mientras que los de Silphidae y Trogidae fueron capturados exclusivamente con la trampa NTP-80, donde los estafilínidos contribuyeron con el 40\% de la abundancia (Cuadro 1).

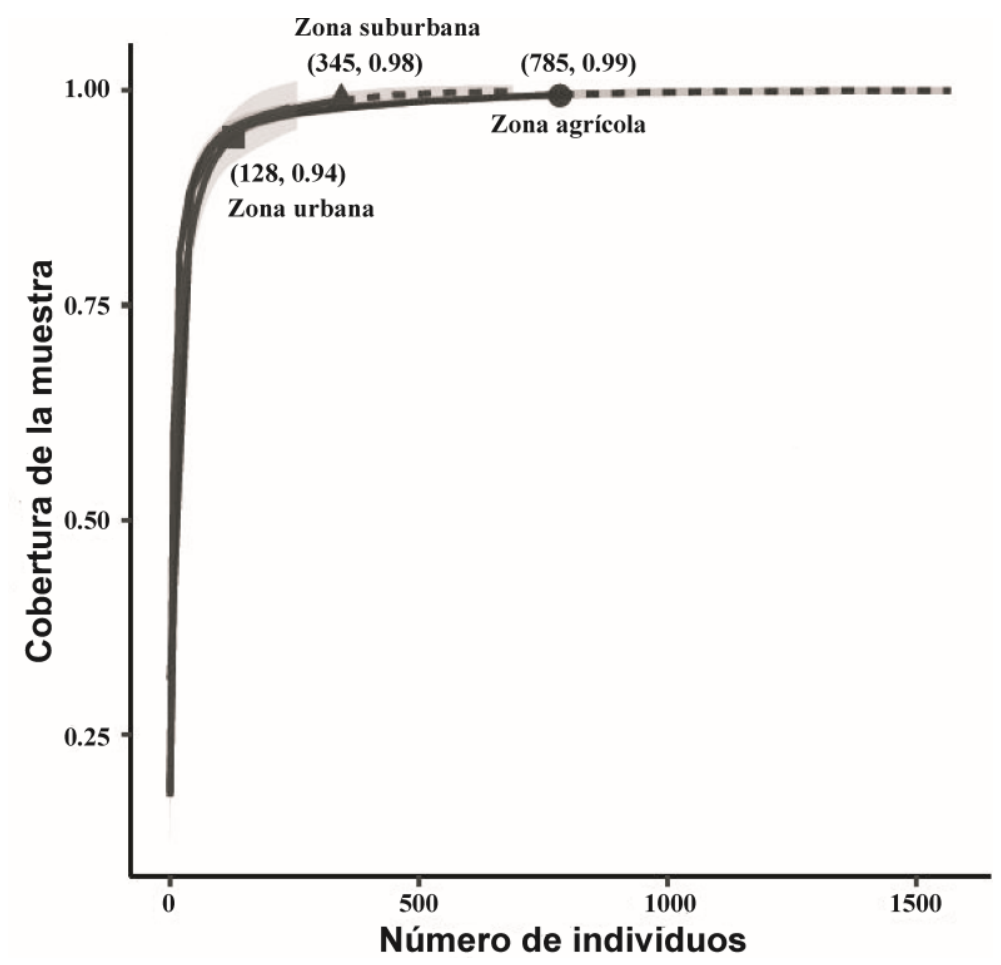

Figura 4. Curva de cobertura de la muestra, para la muestra de referencia (línea continua) y la muestra extrapolada (línea discontinua) de los coleópteros capturados en tres zonas. 
En cuanto a la disimilitud entre los sitios, el valor estimado de diversidad beta total fue de $\beta_{\mathrm{SOR}}=$ $0.347 \mathrm{y}$ el desglose de sus componentes, el recambio espacial $\left(\beta_{\mathrm{SIM}}=0.189\right)$ y el anidamiento $\left(\beta_{\mathrm{NES}}=0.158\right)$ indican que ambos contribuyeron de forma similar a la diversidad beta. El análisis de la diversidad beta a lo largo de los meses del año en cada uno de los sitios, mostró valores muy similares $\left(Z U, \beta_{\text {SOR }}=0.87, \beta_{\text {SIM }}=\right.$ $\left.0.62, \beta_{\mathrm{NES}}=0.25 ; \mathrm{ZS}, \beta_{\mathrm{SOR}}=0.82, \beta_{\mathrm{SIM}}=0.58, \beta_{\mathrm{NES}}=0.24 ; \mathrm{ZA}, \beta_{\mathrm{SOR}}=0.77, \beta_{\mathrm{SIM}}=0.54, \beta_{\mathrm{NES}}=0.23\right)$, donde se observó que el recambio de especies explica los cambios temporales en la diversidad beta en las tres zonas.

Belonuchus sp. y Nicrophorus mexicanus Mattews, 1888 fueron las especies predominantes en todos los sitios, excepto $N$. mexicanus en la zona urbana, donde fue de las especies menos abundantes (Cuadro 1). El único sitio donde ambas especies estuvieron presentes todo el año fue en la zona agrícola. Belonuchus sp. presentó su mayor pico de abundancia durante las lluvias (agosto) en todos los sitios (Fig. 5) y $N$. mexicanus tuvo su máximo al inicio de la sequía (noviembre) tanto en la zona agrícola (Fig. 5a) como en la zona suburbana (Fig. 5b). Por su parte, Anotylus sp. fue la segunda más abundante en la zona urbana después de Belonuchus sp. (Cuadro 1), ambas especies se registraron durante ocho meses en este sitio (Fig. 4c).

La frecuencia de los hábitos alimentarios de las especies fue diferente entre las localidades $\left(X^{2}=\right.$ $16.81 ; P=0.002)$. Los depredadores y los necrófagos fueron más frecuentes en la zona agrícola y en la zona suburbana, los necrófagos también lo fueron en la zona urbana junto con los saprófagos (Cuadro 2).

Cuadro 2. Frecuencias de los hábitos alimentarios en las localidades y método de recolección.

\begin{tabular}{lcccccc}
\hline \multirow{2}{*}{ Hábitos } & \multicolumn{2}{c}{ Zona agrícola } & \multicolumn{2}{c}{ Zona suburbana } & \multicolumn{2}{c}{ Zona urbana } \\
\cline { 2 - 7 } & NTP-80 & Pitfall & NTP-80 & Pitfall & NTP-80 & Pitfall \\
\hline Depredador & 72 & 38 & 39 & 29 & 21 & 18 \\
Micófago & 0 & 18 & 0 & 10 & 0 & 5 \\
Necrófago & 57 & 0 & 37 & 0 & 4 & 0 \\
Saprófago generalista & 1 & 11 & 3 & 0 & 8 & 12 \\
Telio-necrófago & 4 & 0 & 3 & 0 & 1 & 0 \\
\hline
\end{tabular}

Cuadro 3. Especies indicadoras para cada localidad por medio del análisis IndVal. Los valores en negritas indican diferencias significativas. $\mathrm{P}>0.01 *, \mathrm{P}>0.001 * *, \mathrm{P}>0.001 * * *$.

\begin{tabular}{|c|c|c|c|c|c|c|c|}
\hline Localidad & Especie & IndVal & P. value & Localidad & Especie & IndVal & P. value \\
\hline \multirow{16}{*}{$\begin{array}{l}\text { Cultivo } \\
\text { Opuntia }\end{array}$} & N. mexicanus & 0.408 & $0.001 * * *$ & \multirow{7}{*}{$\begin{array}{l}\text { Zona } \\
\text { Urbana }\end{array}$} & Anotylus sp. & 0.317 & $0.001 * * *$ \\
\hline & T. graniger & 0.216 & $0.007 * *$ & & Neohypnus sp. & 0.160 & 0.091 \\
\hline & Belonuchus sp. & 0.337 & $0.030 *$ & & Philonthus sp. & 0.137 & 0.186 \\
\hline & B. erichsoni & 0.229 & 0.023* & & Apocellus sp. & 0.091 & 0.268 \\
\hline & Bryoporus sp. & 0.184 & $0.024 *$ & & Coproporus sp. & 0.091 & 0.305 \\
\hline & Baeocera sp. & 0.225 & 0.067 & & Tachyporinae sp. & 0.091 & 0.258 \\
\hline & Philonthus sp.1 & 0.166 & 0.276 & & Pselaphinae sp.1 & 0.091 & 0.271 \\
\hline & Scydmaeninae sp.1 & 0.160 & 0.188 & \multirow{9}{*}{$\begin{array}{c}\text { Zona } \\
\text { Arqueológica }\end{array}$} & & & \\
\hline & C. maxillosus & 0.134 & 0.629 & & B. ephippiatus & 0.189 & 0.198 \\
\hline & Philonthus sp.5 & 0.110 & 0.331 & & T. truncatus & 0.169 & 0.406 \\
\hline & Scaphidium sp. & 0.108 & 0.631 & & Pselaphinae sp. & 0.138 & 0.492 \\
\hline & Trox plicatus & 0.102 & 0.758 & & Echiaster sp. & 0.137 & 0.161 \\
\hline & Philonthus sp. 2 & 0.078 & 1.000 & & O. discicolle & 0.114 & 0.189 \\
\hline & Philonthus sp. 3 & 0.078 & 1.000 & & Philonthus sp.6 & 0.081 & 0.643 \\
\hline & Suniocharis sp. & 0.078 & 1.000 & & Deroderus sp. & 0.058 & 1.000 \\
\hline & Heterothops sp. & 0.067 & 0.960 & & Platydracus sp. & 0.058 & 1.000 \\
\hline
\end{tabular}


Las especies N. mexicanus, Thanatophilus graniger (Chevrolat, 1883), Belonuchus sp., B. erichsoni Bernhauer, 1917 y Bryoporus sp. tuvieron mayor afinidad por la zona agrícola, en tanto que, Anotylus sp. fue la única especie significativa para la zona urbana y no hubo ninguna para la zona suburbana (Cuadro 3).
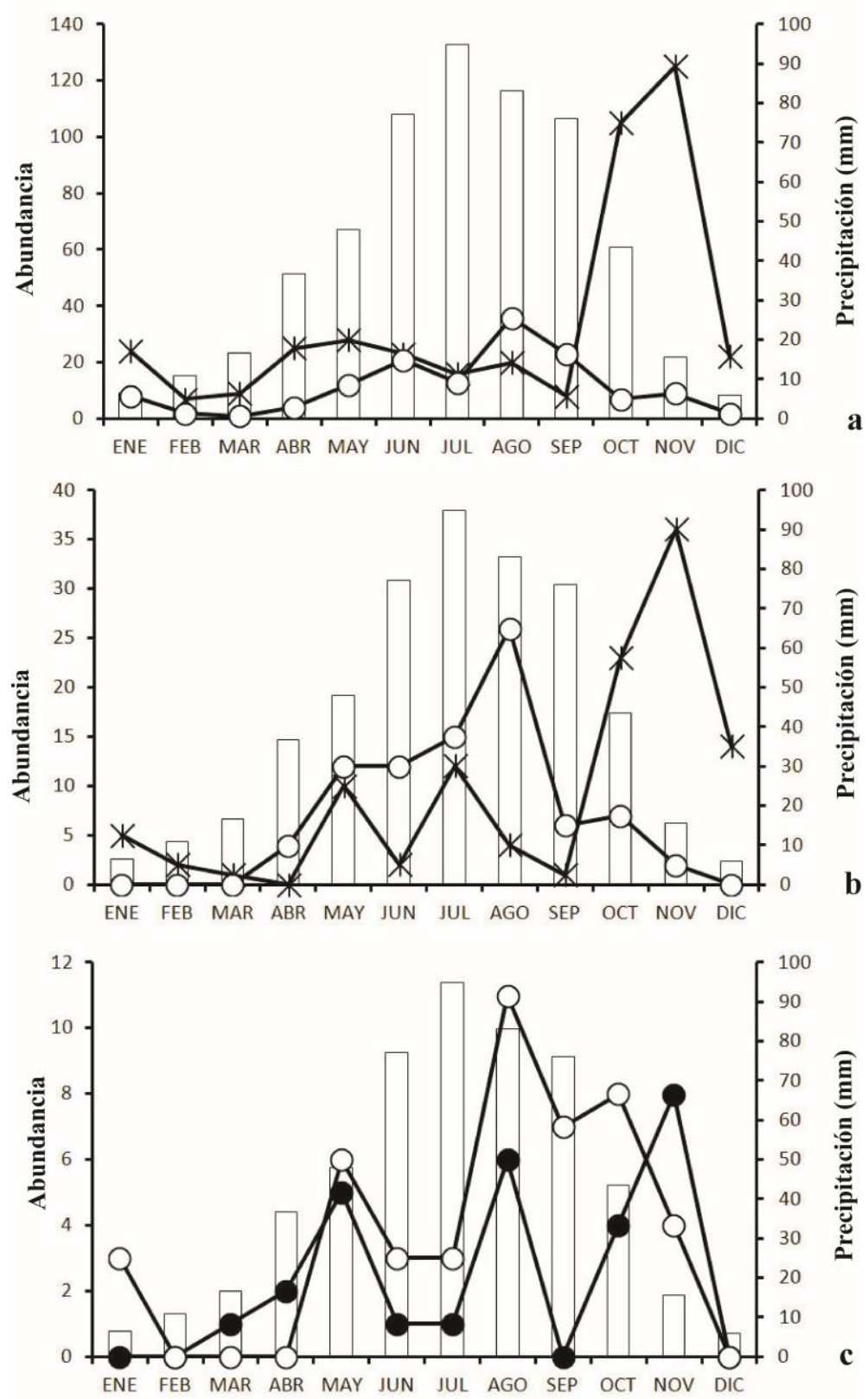

Figura 5. Abundancia mensual de las especies dominantes: N. mexicanus (asterisco), Belonuchus sp. (círculo blanco), Anotylus sp. (circulo negro), y su relación con la precipitación (barras) en los diferentes usos de suelo. a) Zona agrícola. b) Zona suburbana. c) Zona urbana. 


\section{DISCUSIÓN}

La riqueza obtenida para las familias necrófilas estudiadas en la región de Teotihuacán, Estado de México, es alta comparada con la región semiárida de Zapotitlán, las especies de la familia Staphylinidae y las de Silphidae capturadas con la NTP-80 corresponden aproximadamente con el doble de las registradas, respectivamente, para cada una de estas familias en Zapotitlan de las Salinas, Puebla (Jiménez-Sánchez et al., 2013), probablemente debido al proceso de antropización. Es importante considerar que Zapotitlán pertenece a la Reserva de la Biosfera Tehuacan-Cuicatlán ubicada a una altitud de 1,500 m, donde la vegetación predominante es el matorral xerófilo con varios tipos de asociaciones vegetales en una extensión muy amplia y bien conservada. Mientras que en esta zona del Estado de México, aproximadamente tres cuartas partes de la vegetación ha sido alterada para uso agrícola, lo que favorece posiblemente la presencia de especies con un mayor umbral de tolerancia y que se podrían adaptar bien a cualquier tipo de condiciones, como es el caso de C. maxillosus (Staphylinide), T. plicatus (Trogidae) y N. mexicanus (Silphidae), las cuales tienen una distribución geográfica amplia (Vaurie, 1955; Navarrete-Heredia et al., 2002; NavarreteHeredia, 2009). Asimismo, todas las especies de Silphidae, con excepción de T. graniger que es endémica de México y que se distribuye exclusivamente en localidades del Eje Neovolcánico, las otras tres especies están ampliamente distribuidas en México y algunas de ellas hasta Centro y Sudamérica, en varios tipos de vegetación y en un rango altitudinal amplio (Navarrete-Heredia, 2009). La alta riqueza de especies de la familia Silphidae en la zona, también se vio favorecida por la altitud, debido a que la mayor riqueza se obtiene generalmente a elevaciones mayores a 1,700 m (Halffter et al., 1995).

En cuanto al inventario de especies para el estado, se amplió la distribución de Belocnuchus erichsoni, de la cual solo se tenían registros previos de los estados de Querétaro (Navarrete-Heredia et al., 2002) e Hidalgo (Márquez, 2004; Asiain et al., 2011).

Por otro lado, el uso de suelo tuvo un efecto significativo en los parámetros de abundancia, riqueza y diversidad, de los cuales la zona agrícola destacó de manera significativa. En ambientes áridos la disponibilidad de agua en la estación seca es un factor fundamental que contribuye en la distribución de las poblaciones animales y vegetales, además las plantas con menor estrés hídrico pueden albergar una mayor diversidad y abundancia de insectos (Wenninger \& Inouye, 2008), debido a que el riego suplementario incrementa la productividad en los hábitats xéricos (Shochat et al., 2004). Los coleópteros epigeos son grupos importantes de invertebrados en ambientes agrícolas en términos de riqueza y abundancia (Obrtel, 1968), por lo que al ser la actividad agrícola el uso de suelo que ocupa la mayor extensión del territorio estudiado, conformada por un mosaico de parcelas dedicadas además del cultivo de Opuntia, al maíz ( $Z$. mays), el frijol ( $P$. vulgaris) y la acelga (B. vulgaris), entre otros, algunos de ellos dotados de sistemas de riego que aportan humedad durante gran parte del año, fueron probablemente factores que favorecieron el desarrollo de las comunidades de coleópteros necrófilos y epigeos estudiados. De hecho, la zona agrícola fue el único sitio donde las especies necrófilas dominantes (N. mexicanus y Belonuchus sp.) persistieron todo el año.

En general, la riqueza de especies necrófilas mostró un decremento en la zona urbana y suburbana; las especies presentes en estos sitios correspondieron principalmente con especies generalistas (euritópicas), con mayor capacidad de adaptarse a los ambientes perturbados por el humano. Jiménez-Sánchez et al. (2013) encontraron que los estafilínidos más tolerantes a los cambios del hábitat fueron aquellos atraídos a la carroña en zonas urbanas de una región semiárida de Puebla, México. Una disminución de las especies especialistas de bosques en las zonas urbanas se ha observado en casi todos los estudios en gradientes de áreas urbanas a áreas rurales, mientras que las especialistas de los hábitats abiertos se ven favorecidas por las prácticas de manejo a las que son sometidas las áreas urbanas (Deichsel, 2006). Con relación a la comunidad de coleópteros que se alimentan de carroña, como es el caso de la familia Silphidae, se ha visto que su diversidad decrece en función de la pérdida y fragmentación del bosque en un gradiente urbano-rural (Wolf \& Gibbs, 2004) y son más exitosos en bosques extensos que en los pequeños fragmentos generados 
por la urbanización, principalmente debido a que en éstos el procesamiento de cadáveres es principalmente realizado por vertebrados (Trumbo \& Bloch, 2000).

La riqueza de estafilínidos epígeos fue menor en la zona suburbana, esto se debió probablemente a que en esta zona la remoción periódica de plantas, troncos y hojarasca forma parte del mantenimiento de los monumentos arqueológicos (Torres, 2001, E. Jiménez, obs. pers.), lo cual pudo afectar de manera considerable a la comunidad de coleópteros de esta zona. Bohac (1999) observó que, en parques urbanos con manejo deficiente, la estructura ecológica de las comunidades de estafilínidos, es más parecida a la de los bosques seminaturales ubicados en la vecindad de las áreas urbanas.

Con respecto a la diversidad, a pesar de que la zona agrícola tuvo mayor riqueza de especies, fue significativamente menos diversa que la zona urbana y suburbana, las cuales tuvieron la misma riqueza. La zona urbana, de acuerdo con la diversidad del orden $\mathrm{q}=1$, fue 1.1 veces más diversa que la zona suburbana, y esta a su vez 1.3 veces más diversa que la zona agrícola, incluso, la zona urbana fue 1,5 veces más diversa que la zona agrícola. La baja diversidad en la zona agrícola se debe a que aquí se encontraron dos especies (N. mexicanus y Belonuchus sp.) dominantes, que representaron el $70 \%$ de los individuos recolectados en esa zona, mientras que en las otras dos zonas, las especies tuvieron mayor equidad, sobre todo en la zona urbana donde el $90 \%$ tuvieron una abundancia menor de diez individuos, aunque cabe mencionar que fue la zona donde el muestreo fue más incompleto de acuerdo con los valores de cobertura de la muestra.

El hecho de que la proporción de especies raras en la zona urbana fuera mayor que aquella de ambientes no urbanizados, es un patrón que ha sido observado en estafilínidos necrófilos y coprófilos en ambientes abiertos como pastizal vs bosque continuo y fragmentado (Caballero et al., 2009); para coleópteros necrófilos (Scarabaeidae, Trogidae y Silphidae) en un bosque de Pinus-Quercus vs bosque afectado por la tala (Trevilla-Rebollar et al., 2010) o con coleópteros epigeos en ambientes con complejidad estructural baja $v s$ sitios con complejidad alta (Lassau et al., 2005), esto sugiere que los ambientes perturbados parecen tener una comunidad de coleópteros caracterizada por pocas especies dominantes.

En cuanto a la distribución temporal, se encontró que hubo un alto recambio de especies $\left(\beta_{\mathrm{SOR}}\right)$ a lo largo de los meses del año en cada uno de los sitios. De manera particular, la riqueza y la abundancia de coleópteros fueron mayores en la zona agrícola, principalmente durante las lluvias. La familia Staphylinidae mostró una preferencia por esta época del año; este patrón ha sido observado en fauna necrófila en otras zonas de México (Márquez, 2003; Caballero et al., 2009; Trevilla-Rebollar et al., 2010), lo que indica que el régimen de lluvias es un periodo de alta actividad para los estafilínidos. En el caso de los sílfidos representados por la especie $N$. mexicanus, la cual suele ser encontrada prácticamente durante todo el año con un predominio hacia la época de lluvias en varias localidades de México (Navarrete-Heredia, 2009), en el área de estudio se observó que sus poblaciones no disminuyeron durante la época seca, debido a que al tratarse de ambientes antrópicos existe un aporte constante de varios tipos de materia orgánica en descomposición como parte de los restos generados.

El recambio espacial y el anidamiento contribuyeron de manera similar en la variación de la composición del ensamble de especies entre los sitios. El valor de la diversidad beta fue bajo, y probablemente se debido a que la mitad de las especies (17) tuvieron una distribución amplia presentes en los tres sitios, de los cuales la zona agrícola albergó 24 especies que estuvieron presentes tanto en la zona urbana como en la zona suburbana, o al menos en alguna de éstas, y un total de nueve especies fueron exclusivas de alguna de las zonas. Las especies ampliamente distribuidas mostraron una disminución de sus poblaciones en la zona suburbana y la zona urbana. Esto fue evidente para algunas especies depredadoras de Staphylininae tales como: B. erichsoni, Belonuchus sp., Creophilus maxillosus (Linnaeus, 1758) y Philonthus sp.1 o las saprófagas de Silphidae: N. mexicanus y Thanatophilus truncatus (Chevrolat, 1883) (necrófagas), Scaphidiinae: Scaphidium sp. y Baeocera sp. (micófagas) y Oxytelinae: Anotylus sp. 
(saprófaga generalista). Jennings y Tallamy (2006) encontraron que los grupos tróficos más abundantes (depredadores y los saprófagos) fueron los más afectados por la fragmentación de los bosques.

Aún cuando se ha visto que las especies depredadoras están menos especializadas, son muy activas y utilizan una amplia variedad de hábitats para moverse en busca de sus presas (Lassau et al., 2005), se ha observado que la especie B. ephippiatus (Staphylininae), ha sido comúnmente recolectada en cactáceas en descomposición de los géneros Opuntia, Cereus, Agave y otros restos de plantas en putrefacción (Smetana, 1995), por lo que es muy probable que esté especializada en presas que habitan en este tipo de recursos. Sin embargo, en los sitios de muestreo también fue atraída por la carroña, lo cual también ha sido observado en otras zonas semiáridas (Jiménez-Sánchez et al., 2013); asimismo, en el área de estudio fue activa en el suelo, aunque en menor proporción, como lo demuestra la captura de pocos individuos en la trampa de caída. Esta especie estuvo ausente en la zona urbana, donde la presencia de cactáceas fue poco frecuente. Por lo tanto, los hábitos alimentarios es uno de los factores que permiten tratar de explicar la preferencia de las especies por los sitios, a través de los patrones de respuesta en la relación presa-depredador, el grado de especialización de la dieta y la posible asociación de los escarabajos con microhábitats específicos que pueden servir como hábitat o sustrato para su fuente de alimento (Klimaszewski et al., 2013).

Finalmente, aunque se desconoce mucho sobre las variables físicas, químicas y biológicas que determinan la presencia-ausencia o el número de individuos de las especies, las cuales son condiciones necesarias para obtener de manera indirecta ciertas características específicas del hábitat y de esta manera poder considerar a estas especies como buenas indicadoras (Dufrene \& Legendre, 1997), en el presente estudio se encontró que, con base en su abundancia y predominancia en los sitios, la zona agrícola tuvo un mayor número de especies con una asociación alta con este hábitat que con la zona urbana y suburbana, lo que coincide con la mayor productividad observada en la zona, debido al manejo de los cultivos, además de haber albergado a más de la mitad de las especie presentes en los otros dos sitios. Caballero y Cortés (2012) encontraron un mayor número de especies indicadoras de coleópteros en bosques no fragmentados y sitios de cañadas que aparentemente funcionan como refugio de varias especies.

\section{CONCLUSIONES}

Del mosaico de uso de suelo presente en esta región del Estado de México, el dedicado a la agricultura favoreció una mayor abundancia y riqueza, y menor diversidad de coleópteros, debido al aporte constante de materia orgánica y humedad derivada del manejo agrícola, lo cual provocó que las poblaciones de algunas especies de sílfidos y estafilínidos fueran muy altas en esta zona disminuyendo con ello su diversidad, la cual se incrementó en la transición a la zona suburbana y urbana, estas última tuvieron igual riqueza y mayor equidad. El recambio espacial y el anidamiento contribuyeron de manera similar en la variación de la composición del ensamble de especies entre los sitios, la zona agrícola albergo el $70.6 \%$ de las especies que estuvieron presentes tanto en la zona urbana como en la zona suburbana o en alguna de éstas, así como, un mayor número de especies con afinidad alta por este sitio, por lo que podría decirse que la zona agrícola funciona como refugio de las especies de coleópteros necrófilos y epigeos que se distribuyen en esta región semiárida.

Agradecimientos. A Andrea García por su participación en las recolecciones. A Mónica Chico por la elaboración del mapa. Este estudio fue parcialmente apoyado por el Proyecto Zoología de la División de Investigación y Posgrado de la FES Iztacala, UNAM. 


\section{LITERATURA CITADA}

Arellano, L., Castillo-Guevara, C. (2014) Efecto de los incendios forestales no controlados en el ensamble de escarabajos coprófagos (Coleoptera: Scarabaeidae) en un bosque templado del centro de México. Revista Mexicana de Biodiversidad, 85, 854-865.

DOI:10.7550/rmb.41756

Arellano, L., Favila, M. E., Huerta, C. (2005) Diversity of dung and carrion beetles in a disturbed Mexican tropical montane cloud forest and on shade coffee plantations. Biodiversity and Conservation, 14, 601-615.

DOI: 10.1007/s10531-004-3918-3

Asiain, J., Márquez, J., Bueno-Villegas, J. (2011) The Staphylinidae (Coleoptera) Fauna of Los Marmoles National Park, Hidalgo, Mexico. Coleopterists Bulletin, 65, 393-402.

Balog, A., Markó, V. (2007) Rove beetles (Coleoptera: Staphylinidae) in central european apple and pear orchards comparative studies of species richness, abundance and diversity. Journal of Plant Protection Research, 47, 309-320.

Baselga, A. (2010) Partitioning the turnover and nestedness components of beta diversity. Global Ecology and Biogeography, 19, 134-143.

Bohac, J. (1999) Staphylinid beetles as bioindicators. Agriculture, Ecosystems \& Environment, 74, 357372.

DOI: 10.1016/S0167-8809(99)00043-2

Caballero, U., León-Cortés, J. L. (2012) High diversity beetle assemblages attracted to carrion and dung in threatened tropical oak forests in Southern Mexico. Journal of Insect Conservation, 16, 537-547. DOI: 10.1007/s10841-011-9439-y

Caballero, U., León-Cortes, J. L., Morón-Ríos, A. (2009) Response of rove beetles (Staphylinidae) to various habitat types and change in Southern Mexico. Journal of Insect Conservation, 13, 67-75.

DOI: $10.1007 / \mathrm{s} 10841-007-9121-6$

Chivian, E., Bernstein, A. (2015) ¿Cómo la actividad humana amenaza la diversidad? Pp: 59-126. En: E. Chivian, A. Bernstein (Eds). Preservar la vida: De cómo nuestra salud depende de la biodiversidad. Fondo de Cultura Económica-Comisión Nacional para el Conocimiento y Uso de la Biodiversidad. D.F., México.

Deichsel, R. (2006) Species change in an urban setting-ground and rove beetles (Coleoptera: Carabidae and Staphylinidae) in Berlin. Urban Ecosystems, 9, 161-178.

DOI: $10.1007 / \mathrm{s} 11252-006-8588-3$

Deloya, C., Parra-Tabla, V., Delfín-González, H. (2007) Fauna de coleópteros Scarabaeidae Laparosticti y Trogidae (Coleoptera: Scarabaeoidea) asociados al Bosque Mesófilo de Montaña, cafetales bajo sombra y comunidades derivadas en el Centro de Veracruz, México. Neotropical Entomology, 36, $5-21$.

Derunkov, A. V. (2005) Changes in species diversity of rove beetles (Coleoptera, Staphylinidae) depending on the age of pine plantations in central Belarus. Russian Journal of Ecology, 36, 277-284.

Dufrêne, M., Legendre, P. (1997) Species assemblages and indicator species: the need for a flexible asymmetrical approach. Ecological Monographs, 67, 345-366.

Grandchamp, A. C., Niemelä, J., Kotze, J. (2000) The effects of trampling on assemblages of ground beetles (Coleoptera, Carabidae) in urban forest in Helsinki, Finland. Urban Ecosystems, 4, 321332.

Halffter, G., Favila, M. E., Arellano, L. (1995) Spatial distribution of three groups of Coleoptera along an altitudinal transect in the Mexican Transition Zone and its biogeographical implications. Elytron, 9, 151-185.

Hsieh, T. C., Ma, K. H., Chao, A. (2016) iNEXT: An R package for rarefaction and extrapolation of species diversity (Hill numbers). Methods in Ecology and Evolution, 7, 1451-1456.

DOI: 10.1111/2041-210X.12613

INEGI (Instituto Nacional de Estadística y Geografía) (2010a) Compendio de información geográfica 
municipal 2010 San Martín de las Pirámides México. México. Disponible en: http://www.inegi.org.mx/geo/contenidos/topografia/compendio.aspx (Consultado en septiembre 2017).

INEGI (2010b) Compendio de información geográfica municipal 2010 Teotihuacán México. México. Disponible en: http://www.inegi.org.mx/geo/contenidos/topografia/compendio.aspx (Consultado en septiembre 2017).

INEGI (2017) Mapa digital de México. Disponible en: http://gaia.inegi.org.mx/mdm6/?v=bGF0OjE5LjY5MTg5LGxvbjotOTguODIxNjEsejoxMCxsOm MxMTFzZXJ2aWNpb3N8dGMxMTFzZXJ2aWNpb3N8YzQxOA== (Consultado en septiembre 2017).

Ishitani, M., Kotze, D. J., Niemelä, J. (2003) Changes in carabid beetle assemblages across an urban-rural gradient in Japan. Ecography, 26, 481-489.

DOI: $10.1034 /$ j.1600-0587.2003.03436.x

Jałoszynski, P. (2018) Biology of Acarophagous Scydmaeninae. Pp: 285-298. En: O. Betz, U. Irmler, J. Klimaszewski (Eds). Biology of Rove Beetles (Staphylinidae). Springer. Cham, Switzerland.

Jennings, V. H., Tallamy, D. W. (2006) Composition and abundance of ground-dwelling coleoptera in a fragmented and continuous forest. Environmental Entomology, 35, 1550-1560.

DOI: 10.1603/0046-225X(2006)35[1550:CAAOGC]2.0.CO;2

Jiménez-Sánchez, E., Juárez-Gaytán, O. M., Padilla-Ramírez, J. R. (2011) Estafilínidos (Coleoptera: Staphylinidae) necrófilos de Malinalco, Estado de México. Dugesiana, 18, 73-84.

Jiménez-Sánchez, E., Quezada-García, R., Padilla-Ramírez, J. (2013) Diversidad de escarabajos necrófilos (Coleoptera: Scarabaeidae, Silphidae, Staphylinidae y Trogidae) en una región semiárida del valle de Zapotitlán de las Salinas, Puebla, México. Revista de Biología Tropical, 61, 1475-1491.

Klimaszewski, J., Morency, M. J., Labrie, P., Séguin, A., Langor, D., Work, T., Bourdon, C., Thiffault, E., Paré, D., Newton, A. F., Thayer, M. K. (2013) Molecular and microscopic analysis of the gut contents of abundant rove beetle species (Coleoptera: Staphylinidae) in the boreal balsam fir forest of Quebec, Canada. Zookeys, 353, 1-24.

DOI: $10.3897 /$ zookeys.353.5991

Lassau, S. A., Hochuli, D. F., Cassis, G., Reid, C. A. M. (2005) Effects of habitat complexity on forest beetle diversity: do functional groups respond consistently? Diversity and Distributions, 11, 73-82. DOI: 10.1111/j.1366-9516.2005.00124.x

Márquez, J. (2003) Ecological patterns in necrophilous Staphylinidae (Insecta: Coleoptera) from Tlayacapan, Morelos, Mexico. Acta Zoológica Mexicana (nueva serie), 89, 69-83.

Márquez, J. (2004) Primeros registros estatales de las especies mexicanas de Staphylininae (Coleoptera: Staphylinidae). Acta Zoológica Mexicana (nueva serie), 20, 91-97.

Morón, M. A. (2003) Capítulo 1. Antecedentes. Pp: 11-18. En: M. A. Morón (Ed.). Atlas de los escarabajos de México. Coleoptera: Lamellicornia. Vol. II Scarabaeidae,Trogidae, Passalidae y Lucanidae. Argania editio, Barcelona, España.

Morón, M. A., Terrón, R. A. (1984) Distribución altitudinal y estacional de los insectos necrófilos en la sierra Norte de Hidalgo, México. Acta Zoológica Mexicana (nueva serie), 3, 1-47.

Navarrete-Heredia, J. L. (2009) Silphidae (Coleóptera) de México: Diversidad y distribución. Universidad de Guadalajara, Jalisco, México.

Navarrete-Heredia, J. L., Newton, A. F., Thayer, M. K., Ashe, J. S., Chandler, D. S. (2002) Guía ilustrada para los géneros de Staphylinidae (Coleoptera) de México. Illustrated guide to the genera of Staphylinidae (Coleoptera) of Mexico. Universidad de Guadalajara y CONABIO, Guadalajara, México.

Nichols, E., Larsen, T., Spector, S., Davis, A. L., Escobar, F., Favila, M., Vulinec, K., The Scarabaeinae Research Network. (2007) Global dung beetle response to tropical forest modification and fragmentation: A quantitative literature review and meta-analysis. Biological Conservation, 137, 1-19.

Obrtel, R. (1968) Carabidae and Staphylinidae ocurring on soil surface in lucerne fields (Coleoptera). Acta 
Entomologica Bohemoslovaca, 65, 5-20.

Pohl, G. R., Langor, D. W., Spence, J. R. (2007) Rove beetles and ground beetles (Coleoptera: Staphylinidae, Carabidae) as indicators of harvest and regeneration practices in western Canadian foothills forest. Biological Conservation, 137, 294-307.

R Development Core Team (2019) R: a language and environment for statistical computing. Version 3.5.2. Viena, Austria: R Foundation for Statistical Computing. Disponible en: http://www.R-project.org (Consultado en noviembre 2019).

Roberts, D. W. (2012) Package "labdsv". Disponible en: https://cran.r-project.org/web/ packages/labdsv/labdsv.pdf (Consultado en noviembre 2019).

Rodríguez, W. D., Navarrete-Heredia, J. L., Klimaszewski, J., Guevara, R. (2019) The influence of environmental temperature and humidity on the elevational and temporal distributions of rove beetles (Coleoptera: Staphylinidae) in a Quercus L. forest in Jalisco, Mexico. The Coleopterists Bulletin, 73, 202-224.

DOI: $10.1649 / 0010-065 x-73.1 .202$

Shochat, E., Stefanov, W. L., Whitehouse, M. E. A., Faeth, S. H. (2004) Urbanization and spider diversity: influences of human modification of habitat structure and productivity. Ecological Applications, 14, 268-280.

SMN (Servicio Meteorológico Nacional) (2017) Normales Climatológicas por Estación: Estado de México, Otumba NORM 81-10. Disponible en: http://smn.cna.gob.mx/es/informacionclimatologica-ver-estado?estado=mex (Consultado en julio 2017).

Smetana, A. (1995) Rove beetles of the subtribe Philonthina of America North of Mexico (Coleoptera: Staphylinidae): Classification, phylogeny, and taxonomic revision. American Entomological Institute, USA. 946 pp.

Thayer, M. K. (2005) Staphylinidae Latreille, 1802. Pp: 296-344. En: R. G. Beutel, R. A. B. Leschen (Eds.). Coleoptera, beetles. Volume 1: Morphology and systematics (Archostemata, Adephaga, Myxophaga, Polyphaga partim). Walter de Gruyter. Berlin, Germany.

Trumbo, S. T., Bloch, P. L. (2000) Habitat fragmentation and burying beetle abundance and success. Journal Insect Conservation, 4, 245-252.

Torres, P. (2001) Flora fanerogámica de la zona arqueológica de Teotihuacán, Estado de México. Polibotánica, 12, 57-83.

Trevilla-Rebollar, A., Deloya, C., Padilla-Ramírez, J. R. (2010) Coleópteros necrófilos (Scarabaeidae, Silphidae y Trogidae) de Malinalco, Estado de México, México. Neotropical Entomology, 39, 486495.

Uno, S. Cotton, J., Philpott, S. M. (2010) Diversity, abundance, and species composition of ants in urban green spaces. Urban Ecosystems, 13, 425-411.

Vaurie, P. (1955) A revision of the genus Trox in North America (Coleoptera: Scarabaeidae). Bulletin of the American Museum of Natural History, 106, 1-89.

Wenninger, E. J., Inouye, R. S. (2008) Insect community response to plant diversity and productivity in a sagebrush-steppe ecosystem. Journal of Arid Environments, 72, 24-33.

DOI: 10.1016/j.jaridenv.2007.04.005

Wolf, J. M., Gibbs, J. P. (2004) Silphids in urban forests: Diversity and function. Urban Ecosystems, 7, 371-384. 